\section{Pulsus paradoxus: an underused tool}

\section{G. Bandinelli • A. Lagi • P.A. Modesti}

G. Bandinelli • A. Lagi (凶) Department of Emergency Medicine

S. Maria Nuova Hospital, Florence, Italy e-mail: alfonso.lagi@asf.toscana.it

P.A. Modesti

Clinical Medicine and Cardiology Department of Critical Care Medicine and Surgery University of Florence Viale Morgagni 85, I-50134 Florence, Italy e-mail:pa.modesti@unifi.it

Published online: 31 March 2007

\section{A case of recurrent chest pain with syncope}

\section{G. Bandinelli, A. Lagi}

A 69-year-old man was brought to the emergency unit at 3 a.m. because of severe chest pain. He had no history of heart disease, chest pain, shortness of breath, palpitations, diabetes, hypertension or hyperlipidaemia, and there was no family history of heart disease. Chest pain was still present on admission. There were no electrocardiographic (ECG) changes diagnostic of acute cardiac ischaemia. Blood pressure (BP) was $170 / 100 \mathrm{mmHg}$ in both arms, pulse $72 \mathrm{bpm}$ and regular, no fever, and the respiratory rate was 16 breaths/min. Arterial blood gas analysis was not suggestive of pulmonary embolism, and the chest Xray study was normal. While the patient was in the emergency unit, he had a transient loss of consciousness with BP 80/50 $\mathrm{mmHg}$; the ECG morphology remained unchanged with a sinus rhythm at $74 \mathrm{bpm}$. Within $3 \mathrm{~min}$ the patient's BP spontaneously rose to $140 / 70 \mathrm{mmHg}$, and he became fully alert. The peripheral pulses were $2+$ in the upper and lower extremities bilaterally. At cardiac examination, the heart tones were distant with no murmur, rubs or gallups. At echocardiography, the left ventricular chamber size was normal with an adequate left ventricular performance. Another possible explanation for the chest pain was aortic dissection; however, the aortic root was normal (36 $\mathrm{mm})$ although an anterior and posterior slight pericardial effusion was present. The patient was then sent to the medical ward for further assessment.

The initial cardiac enzymes became available at 6 a.m., and were all in the normal range. At 7 a.m. the patient again complained of severe chest pain, followed by syncope with loss of the sphincter tones. The BP at this time was $80 / 40 \mathrm{mmHg}$. The electrocardiogram showed sinus rhythm $(90 \mathrm{bpm})$ with no signs of myocardial ischaemia. Consciousness returned within few seconds, and full consciousness was achieved after $3 \mathrm{~min}$. The BP recovered over time. A computed tomography scan was then performed to investigate the thoracic aorta. Slight pericardial and bilateral pleural effusions were observed with a normal aorta and no signs of pulmonary thromboembolic disease.

At 11 a.m. a new crisis occurred with syncope, dyspnoea and diaphoresis with no chest pain. The pulse was rhythmic with a tachycardia (119 bpm) and the systolic BP was $85 \mathrm{mmHg}$. Consciousness returned fully within 2-3 min. At recovery systolic BP was $130 \mathrm{mmHg}$, but an additional $40 \mathrm{mmHg}$ of pulsus paradoxus (PP) was found. Transthoracic echocardiography was unchanged. Transoesophageal echocardiography did not reveal any sign evocative of aortic rupture. Nevertheless, owing to the presence of the PP, a possible diagnosis of cardiac tamponade was made together with the decision to alert the cardiac surgery ward, and to operate immediately.

While the patient was being prepared for transfer to the cardiovascular surgery ward, haemodynamic parameters rapidly deteriorated. The jugular veins became swollen, cardiac sounds became soft with a sudden onset of shock. The patient was intubated and ventilated. Blood and bloody clots were removed with pericardiocentesis. At 1.00 p.m. a cardiac arrest occurred. Cardiopulmonary resuscitation was begun. At 2.10 p.m. the patient was in asystole.

Autopsy demonstrated blood in the pericardium (450 $\mathrm{ml}$ ), with blood clots. An aortic dissection inside the pericardium was found, beginning at $0.5 \mathrm{~cm}$ from the aortic valve and extending $7 \mathrm{~cm}$.

\section{Comment}

\section{P.A. Modesti}

This case has relevant implications on the role of the physical examination in clinical decision making because the attending physicians decided to perform exploratory surgery on the basis of pulsus paradoxus (PP), despite the negative results of two specific examinations (echocardiography and computed tomography scan).

Although diagnosis in vivo is made in as low as $15 \%$ of the cases, acute dissection represents the most common lifethreatening aortic disease in clinical practice [1]. When the ascending aorta is involved, Stanford type A, the prognosis is very poor without prompt surgical treatment, because if left untreated, the mortality is $50 \%$ within the first $48 \mathrm{~h}[1,2]$ with a mortality rate as high as $1-2 \% / \mathrm{h}$ [3]. Such a short time window requires continuous re-evaluation of clinical signs, 
physical findings and other investigations useful to build a Bayesian probability of diagnosis avoiding delays in obtaining surgery. However, in this patient the "negative" or "inconclusive" results of imaging studies left the clinician with a diagnostic puzzle to solve.

The patient had no history of ischaemic heart disease, hypertension or abdominal aortic aneurysm. The lack of tachycardia, the spontaneous regression of symptoms, as well as the normal left ventricular chamber size and the adequate left ventricular performance enabled the physicians to exclude a true cardiogenic shock. On the other hand the symmetrical blood pressure (BP) reduction in both arms enabled only exclusion of an involvement of a subclavian artery in acute aortic dissection. During hypotension, which was preceded by no warning, no changes in heart rate were recorded so that a neurally mediated (reflex) syncope, precipitated by severe aortic pain, with a prevalent contribution of vasodilatation rather than bradycardia, might be hypothesised. These findings make unlikely the possibility of an acute myocardial infarction but do not allow one to exclude an aortic dissection.

An acute aortic dissection was not confirmed by transoesophageal echocardiography and aortic spiral computed tomography scan. However, the clinicians were uncomfortable in discarding the diagnosis of aortic dissection because neither test was truly normal. Rather, both studies showed the presence of pericardial and pleural effusions that may accompany aortic dissection. The haemodynamic weight of pericardial effusion and the presence of cardiac tamponade was finally recognised after the third episode of syncope when PP was appreciated. This finding prompted the clinician to proceed to surgery.

The usual definition of PP is an inspiratory fall in systolic BP exceeding $10 \mathrm{mmHg}$. Kussmaul [4] first described the finding as the diminution or complete absence of the radial pulse during inspiration in 3 patients with pericardial disease. It was felt to be a paradox because despite the absence of radial pulses, heart sounds could be auscultated. The term "paradox" is unfortunate because the finding is nothing more than an exaggeration of normal physiologic change $(6 \pm 3$ $\mathrm{mmHg}$ in normal persons) [5]. Therefore, when checking for PP the clinician has to have the patient breathe quietly and regularly, because even normal persons can induce a PP with vigorous respirations.

$\mathrm{PP}$ is detected by palpating the pulse or by using BP measurement (cuff sphygmomanometer). Systolic BP during inspiration and expiration has to be measured. The difference between the two recorded pressures is the PP value. The PP value that appears reproducible after at least three attempts is the one to be considered. Only paradoxical pulses exceeding 15-20 $\mathrm{mmHg}$ are palpable [6] so that the use of BP measurement is preferred by most clinicians [7], with the added advantage of quantifying the finding. The use of other noninvasive devices useful to measure PP has been tested $[8,9]$. In monitored patients, PP can also be noted in the pulse oximetry tracing appearing as respiratory movement of the tracing's baseline [10]. The height of the oscillation correlates with the severity of PP [10].

The clinical significance of PP is high in cardiac tamponade (defined as improvement in cardiac output of $20 \%$ or more following pericardiocentesis) and acute asthma. PP is detectable in $98 \%$ of patients with cardiac tamponade, where it is one of three key findings, the other being elevated neck veins (sensitivity 100\%) and tachycardia (sensitivity $77 \%-100 \%$ ). A compilation of common and not so common physical findings seen in 56 patients with cardiac tamponade diagnosed at the bedside by Guberman et al. [11] is reported in Table 1 . The finding of a PP>12 $\mathrm{mmHg}$ discriminates patients with tamponade with a sensitivity of $98 \%$, specificity of $83 \%$, positive likelihood ratio of 5.9 and negative likelihood ratio of 0.03 [11]. In only $2 \%$ of patients with tamponade, a PP is absent due to one of five associated disorders: (1) atrial septal defect, (2) severe left ventricular dysfunction, (3) regional tamponade (tamponade affecting only one or two heart chambers, a complication of cardiac surgery), (4) severe hypotension and (5) aortic regurgitation. Knowing that aortic regurgitation may eliminate PP is especially significant because patients with proximal type (type A) aortic dissection and haemopericardium may lack the paradoxical pulse, despite significant tamponade, when the aortic valve is involved. The unaware clinician may thus exclude the possibility of tamponade to the harm of the patient. Indeed the patient of Bandinelli and Lagi had no signs of aortic regurgitation at both physical examination and echocardiography.

When the pressure of fluid inside the pericardial space exceeds the diastolic pressure in the heart chambers, tamponade develops. The diastolic BP in cardiac chambers, reflected in the neck veins, then becomes a measurement of the forces acting to compress the heart. The four chambers, now smaller in size, begin to compete for space, and an increase in size of one comes at the expense of another. Inspiration increases the filling of the right side and shifts the interventricular septum to the left thus obliterating the left ventricular chamber. Therefore, PP is absent in cardiac tamponade associated with an atrial septal defect (when the right ventricle does not fill more during inspiration), and in aortic regurgitation (when the left ventricle fills from a different

Table 1 Physical findings identified in 56 patients with cardiac tamponade diagnosed at the bedside [11]

\begin{tabular}{lr} 
& $\%$ \\
\hline Elevated jugular vein pressure & 100 \\
Pulsus paradoxus & 98 \\
Tachypnoea & 80 \\
Tachycardia & 77 \\
Systolic blood pressure $<100 \mathrm{mmHg}$ & 36 \\
Decreased heart sounds & 34 \\
Rub & 29 \\
Rapidly falling blood pressure & 25
\end{tabular}


Table 2 Aetiologies of pulsus paradoxus other than pericardial effusion and cardiac tamponade

Large pulmonary embolus

Severe COPD exacerbation

Bronchial asthma

Tension pneumothorax

Large pleural effusions

source than the left atrium). A regional tamponade, as probably initially occurred in the patient of Bandinelli and Lagi, compresses only one or two chambers, may limit cardiac output, but is too confined to cause the heart chamber to compete for space and to generate a PP. PP is also absent in conditions with fixed cardiac output such as supravalvular, valvular and subvalvular aortic stenosis, mitral valve stenosis, and in conditions with severely impaired left ventricular systolic function such as severe cardiomyopathy or acute myocardial infarction.

PP is not $100 \%$ specific for the diagnosis of pericardial effusion and cardiac tamponade. A list of other pathologic conditions in which PP can be seen is presented in Table 2. In particular, conditions characterised by laboured respiration (asthma) may present with PP. Indeed the initial National Heart, Lung and Blood Institute guidelines for the diagnosis and management of asthma specified that patients with a PP> $12 \mathrm{mmHg}$ deserve hospital admission [12]. Present guidelines recommend PP measurement although do not identify PP thresholds [7]. Traditional teaching in medical schools includes the expectation that physicians have the ability to measure PP with a BP cuff as a useful adjunct to physical examination. However, PP assessment is often considered time-consuming and only rarely are students instructed to practise their skill in determining PP, which thus remains largely underused in the clinical setting.

In conclusion, although the gold standard for the diagnosis of a pericardial effusion is echocardiography, the diagnosis of cardiac tamponade can be based on physical examination. There is a continuum of patients with pericardial effusion, those with a compensated haemodynamic status and those with a decompensated tamponade status. The point at which the compensatory mechanisms fail and tamponade ensues may not be readily identified by echo, but usually can be readily identified by physical examination.

\section{References}

1. Green GR, Kron IL (2003) Aortic dissection. In: Cohn LH, Edmunds LH Jr (eds) Cardiac surgery in the adult, 2nd edn. McGraw-Hill, New York, NY, pp 1095-1122

2. Hagan PG, Nienaber CA, Isselbacher EM et al (2000) The International Registry of Acute Aortic Dissection (IRAD): new insights into an old disease. JAMA 283:897-903

3. Hirst AE Jr, Johns VJ Jr, Kime SW Jr (1958) Dissecting aneurysm of the aorta: a review of 505 cases. Medicine (Baltimore) 37:217-279

4. Kussmaul A (1873) On adhesive mediastino-pericarditis and the paradoxical pulse. Berl Klin Wochenschr 10:433-435

5. Curtiss EI, Reddy PS, Uretsky BF, Cecchetti AA (1988) Pulsus paradoxus: definition and relation to the severity of cardiac tamponade. Am Heart J 115:391-398.

6. Fowler NO (1994) Pulsus paradoxus. Heart Dis Stroke 3:68-69

7. National Heart, Blood, and Lung Institute (1997) National Asthma Education and Prevention Program. Expert Panel Report 2: Guidelines for the Diagnosis and Management of Asthma. National Institutes of Health, Bethesda, MD. National Institutes of Health, Publication no. 97-4051

8. Jay GD, Onuma K, Davis R et al (2000) Analysis of physician ability in the measurement of pulsus paradoxus by sphygmomanometry. Chest 118:348-352

9. Rayner J, Trespalacios F, Machan J et al (2006) Continuous noninvasive measurement of pulsus paradoxus complements medical decision making in assessment of acute asthma severity. Chest 130:754-765

10. Hartert TV, Wheeler AP, Sheller JR (1999) Use of pulse oximetry to recognize severity of airflow obstruction in obstructive airway disease: correlation with pulsus paradoxus. Chest 115:475-481

11. Guberman BA, Fowler NO, Engel PJ, Gueron M, Allen JM (1981) Cardiac tamponade in medical patients. Circulation 64:633-640

12. National Heart, Blood, and Lung Institute (1991) National Asthma Education and Prevention Program. Expert panel report: guidelines for the diagnosis and management of asthma. National Institutes of Health, Bethesda, MD. Publication no. 91-3042 